\title{
Zodiac Calendar and Market Returns
}

\author{
Alex Meisami \\ Judd Leighton School of Business and Economics \\ Indiana University South Bend \\ E-mail: ameisami@iusb.edu
}

Received: May 5, 2013 Accepted: May 22, $2013 \quad$ Published: June 1, 2013

doi:10.5296/ajfa.v5i1.3637 URL: http://dx.doi.org/10.5296/ajfa.v5i1.3637

\begin{abstract}
Throughout history, Chinese zodiac astrology has significantly influenced the way people in that area think, feel, or make daily decisions. In the zodiac calendar, each year is marked by one of twelve symbolic animals and each animal is associated with its own specific traits. Previous literature suggests investors' mood, attitude, and behavior are influenced by natural events such as cloudy or sunny weather, or lunar cycles. Cultural events such as holidays or religious feasts are also shown to have impact on investing decisions. In this paper we study the stock market returns in different lunar years in the zodiac calendar. We find that Hang Seng's mean returns are higher in Rat years and both mean and median returns are lower in Snake years. These results are statistically significant despite a small sample size. For S\&P 500 , only Snake and Rooster years show lower, statistically significant, holding period return. Results are inconclusive for other animal-years or other categorizations in the zodiac astrology.
\end{abstract}

Keywords: Zodiac calendar, Hang Seng, S\&P 500, Stock market returns, Market efficiency 


\section{Introduction}

Previous literature studies the effect of cyclical and natural events on stock returns. Yuan, Zheng, and Zhu (2006) review the impact of lunar phases on stock market returns and determine there is a decline in return of $3 \%$ to $5 \%$ per annum on a seven day window around a full moon, as opposed to days near a new moon. Hirshleifer and Shumway (2003) find a significant correlation between sunshine and stock market returns. In the same vein, Saunders (1993) shows that market returns on cloudy days are lower than the sunny days. Other studies document how mood and behavior of individuals affect stock market returns. Mehran, Meisami, and Busenbark (2012) find that joyous (solemn) mood and demeanor of different Jewish holidays has a positive (negative) effect on the stock market returns. According the Wall Street Journal, in 2013 a number Chinese couples rushed to get married before the lunar year Dragon ended and the lunar year Snake began. This haste was reinforced as the coming year was also a "blind year" (it did not embrace the first day of spring).

In this paper we study market returns in different zodiac years. We hypothesize the market returns vary in each zodiac year based on demeanor and characteristics attributed to that year. We believe this is important since throughout history the zodiac calendar plays a noteworthy role in how Chinese and, to a lesser extent, others make important decisions.

Section 2 presents a brief summary of the meaning behind the years in zodiac calendar. In Section 3, we highlight some of the relevant literature. We present our data, methods, and findings in Section 4 and conclude in section 5.

\section{Zodiac Calendar}

Mythology plays a significant role in the Chinese culture. Chinese zodiac calendar is created and influenced by this Mythology. The zodiac calendar consists of different classes of diverse categories each with different interpretation. The simplest category divides things of this world in two: yin and yang. The two represent traditional way of understanding the world from zodiac point of view. In brief, yin represents darkness, cold, shrinking, and fragile. Yang, on the other hand, represents light, upward, enlarging, lively, hot, and strong. In addition, there are five main zodiac elements: earth, fire, metal, water, and wood. These five elements are related to five main planets in the solar system. According to the zodiac philosophy, all the natural phenomena around us are a direct result of interaction between these five elements. For instance, wood causes fire, water causes wood, water conquers fire, and fire conquers metal. These five elements could also represent direction, color, season, body parts, senses, and tastes.

In addition to the elements, there are twelve animals in the zodiac calendar: rat, ox, tiger, rabbit, dragon, snake, horse, sheep, monkey, rooster, dog and boar. Why were these particular animals included? Ancient myth states these exclusive twelve animals where included because they accepted an invitation. Hence, they were rewarded their own signs on the zodiac calendar. Table 1 summarizes what each of these animals signify. 
Table 1. What does Each Zodiac Animal Symbolize?

\begin{tabular}{ll}
\hline Rat & Sociable, shrewd, charismatic, intense, tenacious \\
Ox & Dependable, calm, ambitious, born leader, hardworking, logical \\
Tiger & Rebellious, unpredictable, sincere, generous, daring, impulsive \\
Rabbit & Kind, gracious, good friend, shy, elegant, reserved \\
Dragon & Strong, proud, eccentric, intellectual, passionate \\
Snake & Deep thinker, creative, prudent, purposeful, wise \\
Horse & Popular, earthly, magnetic, cheerful, agile, magnetic \\
Sheep & Righteous, gentle, compassionate, mothering, peaceful \\
Monkey & Inventor, problem solver, polite, sociable, competitive \\
Rooster & Scientific, organized, decisive, conservative, alert, zealous, practical \\
Dog & Intelligent, honest, loyal, attractive, straightforward, moralistic \\
Boar & Patient, trusting, thoughtful, understanding, sturdy \\
\hline
\end{tabular}

Each animal, which represents one year in a 12-year cycle in the Chinese lunar calendar, is described in details by using the precise knowledge of astronomy that existed at the time. Finally, there is Chinese Feng Shui that is related to the zodiac through the five elements. In short, the Chinese Zodiac employs the positioning of celestials along with the interaction of the five elements, yin and yang, the twelve animals and the ten heavenly stems. There are two heavenly stems for each of the five elements, a yin and a yang.

\section{Literature Review}

Ariel (1990) and Sullivan, Timmermann, and White (2001) study the connection between holidays and stock market returns. They find on the days immediately preceding an American holiday, the returns are about $0.38 \%$ greater in an equal-weighted index and even greater in a value-weighted index. Ariel concludes that increased stock returns, near American holidays, is caused neither by other previously researched effects- such as the January-effect or weekend-effect- nor by market specialists since positive returns on these days occur well prior to the market close, and there is miniscule evidence of a discernable ask/bid component. To explain this, he hypothesizes that short sellers may desire to close risky positions prior to the holiday; nevertheless, this is determined to be somewhat inconclusive. Analysis as to if the mood of the American holiday had an impact on the returns is not presented by these authors.

Investors' mood (not specific to holidays) and its impact on stock market returns is studied by Hirshleifer and Shumway (2003). By reviewing market returns on sunny days - days widely linked with good moods - they are able to show sunshine is correlated with increased stock market returns. By observing traders' behavior, they find that investors would marginally benefit from trading on sunny days. However, after including transaction costs and controlling for additional variables, their findings are uncertain concerning the extent to which sunshine-based trading is beneficial. Hirschliefer and Shumway (2003) provide evidence that investors are subject to various conscious and subconscious mood biases when 
making investment decisions, suggesting that investors should be cognizant of their moods when trading stocks.

Similarly, an analysis of stock market returns based on lunar phases is documented by Yuan, Zheng, and Zhu (2006). The authors review lunar phases and stock market returns and determine a 3-5 percent deduction in return, per annum, on a seven day window around the full moon relative to return in the same window surrounding the new moon.

\section{Data, Methodology, and Empirical Results}

We used Yahoo Finance to extract the S\&P 500 (S\&P hereafter) adjusted daily closing index returns for the period 1950-2011. We used Bloomberg to download Hang Seng index (HSI hereafter) values. Our data range for HSI is 1964-2013; there is no data available before 1964. Next, we merged this data with zodiac calendar data for the same period. The zodiac calendar is based on lunar phases therefore it rotates through the Gregorian calendar. Our first task was to detect the start and the end date for each zodiac year in the Gregorian calendar. Second, we kept the S\&P values for the first 10 days and the last 10 days of each zodiac year. Third, we calculated the average price for the first 10 trading days and the last 10 trading days of the each zodiac year. Finally, we calculated the holding period return (HPR) for each zodiac year as:

$H P R=[($ average price for the last 10 trading days of the zodiac lunar year)/(average pricefor the first 10 trading days of the zodiac lunar year)] -1 .

Our final sample includes 62 zodiac years from $1 / 3 / 1950$ to $2 / 22 / 2011$. For HSI we use a slightly different methodology. HSI has missing values around the Chinese New Year making it less useful to use the previous method to calculate the HPR. In this case, we calculate the average index value for the first and last month of the lunar calendar. Then the HPR is simply calculated as:

HPR Hang Seng= (average index value in the last month of the zodiac lunar calendar) average index value in the first month of the zodiac lunar calendar)-1.

In Table 2 we calculate the HPRs separately for yin and yang years. As mentioned before, in the Chinese zodiac calendar, yin is interpreted as dark, inactive, sliding, cold, shrinking, and weak; yang is interpreted as active, clear, rising, hot, and sturdy. For HSI, there are 24 yin and 24 yang years in our sample. Mean (median) HPR is $19.98 \%(16.42 \%)$ for the yin years and $24.73 \%(18.55 \%)$ for the yang years. For S\&P there are 31 yin and 31 yang years. Mean (median) HPR is $7.81 \%(8.75 \%)$ for the yin and $8.19 \%(10.43 \%)$ for the yang years. Previous studies show positive relation between stock returns and feeling good; consistently, yang years seem to have higher mean (median) returns. The difference, as indicated by the t-test, is not significant. We also calculate the p-value using the Wilcoxon-Mann-Whitney test for medians; once again, there is no significant difference between the medians. Although statistically insignificant, the mean median difference between yin and yang years returns are higher for the HSI compared to S\&P. Overall, the results are somewhat consistent with the notion that the yang years, that are associated with good feelings, lead to higher market returns. Also, the results indicate that the HSI investors care more about the meanings of the 
yin and yang years as opposed to the S\&P investors, consistent with the view that local culture and norms have a more significant impact on their own local index than on foreign indices.

Table 2. Yin, Yang, and Overall Return Statistics

Panel A. Hang Seng

\begin{tabular}{llllc}
\hline & Mean & Median & StDev & Occurrence \\
\hline All & $22.41 \%$ & $18.13 \%$ & $57.26 \%$ & 48 \\
Yin & $19.98 \%$ & $16.42 \%$ & $70.30 \%$ & 24 \\
Yang & $24.73 \%$ & $18.55 \%$ & $40.95 \%$ & 24 \\
Diff. $t \& p$-values & $(0.7798)$ & $(0.8828)$ & & \\
\hline
\end{tabular}

Panel B. S\&P 500

\begin{tabular}{llllc}
\hline & Mean & Median & StDev & Occurrence \\
\hline All & $8.00 \%$ & $9.89 \%$ & $14.79 \%$ & 62 \\
Yin & $7.81 \%$ & $8.75 \%$ & $14.13 \%$ & 31 \\
Yang & $8.19 \%$ & $10.43 \%$ & $15.66 \%$ & 31 \\
Diff. $t \& p$-values & $(0.9207)$ & $(0.2249)$ & & \\
\hline
\end{tabular}

Table 3 presents the market mean and median returns in years concurrent with each of the five zodiac years earth, fire, metal, water, and wood. The $f$ and $p$-values are the result of the ANOV test performed to evaluate the difference between the means of each group. For HSI there are 10 observations for Earth, Fire, and Metal, 9 observations for Water, and 8 observations for Wood. The mean (median) HPR is equal to $44.09 \%(18.63 \%)$ for Water. Earth has the largest median. In the Chinese culture, it is believed that individuals born in Earth and Water lunar-years are more likely to respond to circumstances.

For S\&P (panel B) there are 13 observations for earth and metal and 12 observations for fire, water, and wood. We do not find similar results for S\&P; however, overlooking the statistical significance, the Wood years show the largest mean and median returns. 


\section{Macrothink}

Table 3. Five Zodiac Elements and Market Returns

Panel A: Hang Seng

\begin{tabular}{llllc}
\hline Five Elements & Mean & Median & StDev & Occurrence \\
\hline Earth & $32.86 \%$ & $40.33 \%$ & $39.64 \%$ & 10 \\
Fire & $6.03 \%$ & $4.47 \%$ & $23.50 \%$ & 10 \\
Metal & $16.50 \%$ & $18.40 \%$ & $32.65 \%$ & 10 \\
Water & $44.09 \%$ & $18.98 \%$ & $113.62 \%$ & 9 \\
Wood & $12.81 \%$ & $19.27 \%$ & $37.38 \%$ & 8 \\
$f$-value & $(0.67)$ & & & \\
$p$-value & $(0.6141)$ & & & \\
\hline
\end{tabular}

Panel B: S\&P 500

\begin{tabular}{llllc}
\hline Five Elements & Mean & Median & StDev & Occurrence \\
\hline Earth & $9.56 \%$ & $12.67 \%$ & $18.39 \%$ & 13 \\
Fire & $4.17 \%$ & $0.75 \%$ & $12.22 \%$ & 12 \\
Metal & $6.92 \%$ & $9.47 \%$ & $11.64 \%$ & 13 \\
Water & $6.08 \%$ & $7.66 \%$ & $16.09 \%$ & 12 \\
Wood & $13.24 \%$ & $13.63 \%$ & $15.31 \%$ & 12 \\
$f$-value & $(0.67)$ & & & \\
$p$-value & $(0.6180)$ & & & \\
\hline
\end{tabular}

According to the Chinese zodiac philosophy, each of the five elements can manifest in a yin or yang form. This leads to 10 groups. These 10 groups are called Heavenly Stems. Table 4 shows the HPRs for these ten groups. The $f$ and $p$-values are the result of the ANOVA test performed to evaluate the difference between the means of each group. Excluding yin Earth and yang Metal with 7 observations, the other 8 groups have 6 observations each. 
Table 4. Ten Heavenly Stems and Market Returns

Panel A: Hang Seng

\begin{tabular}{llllc}
\hline Heavenly Stem & Mean & Median & StDev & Occurrence \\
\hline Yin Earth & $47.22 \%$ & $46.76 \%$ & $35.49 \%$ & 5 \\
Yin Fire & $-8.89 \%$ & $-9.18 \%$ & $17.09 \%$ & 5 \\
Yin Metal & $7.43 \%$ & $-10.08 \%$ & $37.60 \%$ & 5 \\
Yin Water & $20.27 \%$ & $28.62 \%$ & $67.56 \%$ & 4 \\
Yin Wood & $37.43 \%$ & $39.55 \%$ & $22.07 \%$ & 4 \\
Yang Earth & $18.51 \%$ & $27.64 \%$ & $41.96 \%$ & 5 \\
Yang Fire & $20.95 \%$ & $18.13 \%$ & $19.84 \%$ & 5 \\
Yang Metal & $25.56 \%$ & $22.22 \%$ & $27.94 \%$ & 5 \\
Yang Water & $63.14 \%$ & $18.98 \%$ & $146.20 \%$ & 5 \\
Yang Wood & $-11.81 \%$ & $-14.10 \%$ & $34.01 \%$ & 4 \\
$f$-value & $(0.74)$ & & & \\
$p$-value & $(0.6662)$ & & & \\
\hline
\end{tabular}

Panel B: S\&P 500

\begin{tabular}{llllc}
\hline Heavenly Stem & Mean & Median & StDev & Occurrence \\
\hline Yin Earth & $9.33 \%$ & $12.67 \%$ & $12.81 \%$ & 7 \\
Yin Fire & $-0.22 \%$ & $-4.78 \%$ & $11.73 \%$ & 6 \\
Yin Metal & $2.09 \%$ & $9.11 \%$ & $13.15 \%$ & 6 \\
Yin Water & $9.03 \%$ & $9.45 \%$ & $17.47 \%$ & 6 \\
Yin Wood & $18.59 \%$ & $19.30 \%$ & $11.17 \%$ & 6 \\
Yang Earth & $9.84 \%$ & $12.60 \%$ & $24.79 \%$ & 6 \\
Yang Fire & $8.56 \%$ & $7.42 \%$ & $12.03 \%$ & 6 \\
Yang Metal & $11.06 \%$ & $10.43 \%$ & $9.14 \%$ & 7 \\
Yang Water & $3.13 \%$ & $6.42 \%$ & $15.61 \%$ & 6 \\
Yang Wood & $7.90 \%$ & $8.29 \%$ & $17.95 \%$ & 6 \\
$f$-value & $(0.75)$ & & & \\
$p$-value & $(0.6608)$ & & & \\
\hline
\end{tabular}

For Hang Seng, once again Earth and Water stand out, especially yang Water (in line with what we saw in Table 3) and yin Earth. Yang Water has the highest mean (63.14\%) but a mediocre median; yin Earth however, has the second largest mean $(47.22 \%)$ and the largest median (46.76\%). Yin Wood also stands out with the mean (median) of 37.4\% (39.55\%). Yin Wood has the highest mean and median (18.59\% and 19.30\%) for S\&P also. This is followed by yang Metal with mean of $11.06 \%$ and median of $10.43 \%$. At first glance, other groups have considerably lower mean and medians. However, the $f$ and $p$-values form the ANOVA test do not suggest significant difference between the average HPRs of these 10 groups. This low power of the test is most likely due to the small sample size. The sample size problem is more sever for HSI. 
In Table 5 we calculate HPRs for each of the 12 animals. Each animal represents one zodiac year (see Table 1 for the significance of each animal in the zodiac calendar). For HSI the Rat year has the highest mean $(81.30 \%)$. The Snake year has the lowest mean return of negative $15.79 \%$. Interestingly, year 2013 faced a transition from the Dragon to the Snake lunar year. According to the Wall Street Journal, many individuals in Hong Kong hurried to get married before the Snake arrives.

Panel B shows that the Snake year has a negative return even for S\&P. Sheep has the highest mean (18.97\%) and median (17.54\%) HPR. Again, possibly because of the small sample size, the $f$ and $p$-values do not show statistical significance.

Table 5. Twelve Zodiac Animals and Market Returns

Panel A: Hang Seng

\begin{tabular}{lrrrc}
\hline Year & Mean & Median & StDev & Occurrence \\
\hline Rat & $81.30 \%$ & $22.89 \%$ & $163.14 \%$ & 4 \\
Ox & $-6.43 \%$ & $-2.01 \%$ & $52.89 \%$ & 4 \\
Tiger & $3.96 \%$ & $8.08 \%$ & $38.66 \%$ & 4 \\
Rabbit & $27.77 \%$ & $24.92 \%$ & $47.36 \%$ & 4 \\
Dragon & $15.44 \%$ & $17.02 \%$ & $17.18 \%$ & 4 \\
Snake & $-15.79 \%$ & $-10.13 \%$ & $13.41 \%$ & 3 \\
Horse & $7.40 \%$ & $7.75 \%$ & $20.55 \%$ & 4 \\
Sheep & $36.20 \%$ & $41.62 \%$ & $37.64 \%$ & 4 \\
Monkey & $41.40 \%$ & $45.22 \%$ & $33.82 \%$ & 4 \\
Rooster & $34.94 \%$ & $28.77 \%$ & $44.66 \%$ & 4 \\
Dog & $-1.11 \%$ & $-1.89 \%$ & $36.21 \%$ & 4 \\
Boar & $34.26 \%$ & $34.28 \%$ & $22.71 \%$ & 4 \\
$f$-value & $(0.78)$ & & & \\
p-value & $(0.6557)$ & & & \\
\hline
\end{tabular}

Panel B: S\&P 500

\begin{tabular}{lrrrc}
\hline Year & Mean & Median & StDev & Occurrence \\
\hline Rat & $3.74 \%$ & $10.43 \%$ & $23.17 \%$ & 5 \\
Ox & $9.72 \%$ & $13.18 \%$ & $15.96 \%$ & 6 \\
Tiger & $10.41 \%$ & $19.47 \%$ & $18.89 \%$ & 6 \\
Rabbit & $10.75 \%$ & $12.67 \%$ & $11.58 \%$ & 5 \\
Dragon & $5.38 \%$ & $7.66 \%$ & $7.32 \%$ & 5 \\
Snake & $-1.69 \%$ & $0.84 \%$ & $13.18 \%$ & 5 \\
Horse & $5.60 \%$ & $8.64 \%$ & $21.19 \%$ & 5
\end{tabular}

\footnotetext{
1 "Jacky Choi, who is in his mid-30s, hurried to get married just before the Lunar New Year-in part because he wanted to avoid having a wedding during the Year of the Snake." The Year of the Snake Draws Hisses-and Yawns. By Te-Ping Chen and Fiona Law. Walls Street Journal- February $7, \quad 2013$. http://online.wsj.com/article/SB10001424127887324590904578288182480182760.html
} 


$\begin{array}{lrrrr}\text { Sheep } & 18.97 \% & 17.54 \% & 10.63 \% & 5 \\ \text { Monkey } & 7.41 \% & 5.19 \% & 5.63 \% & 5 \\ \text { Rooster } & -2.68 \% & -4.84 \% & 9.25 \% & 5 \\ \text { Dog } & 16.19 \% & 14.00 \% & 13.81 \% & 5 \\ \text { Boar } & 11.44 \% & 8.75 \% & 15.31 \% & 5 \\ \text { f-value } & (0.94) & & & \\ \text { p-value } & (0.5135) & & & \end{array}$

Next, we define a dummy variable for each zodiac animal. For example, a zodiac year is assigned the value 1 if it falls in the Rat year, and 0 otherwise. Then, by using the following OLS regression, we investigate if HSI holding period returns are affected by a specific animal-year.

$$
H P R_{\text {Hang Seng }}=\alpha+\beta \text { (animal_year_dummy) }
$$

Results are presented in Table 6. Rat year coefficient is significant at 5\% level (p-value is .0299); however the test fails to show similar results for the Snake or other animal-years.

Table 6. OLS Regression of Year-Dummies over Hang Seng Returns

\begin{tabular}{lrrr}
\hline & $\begin{array}{r}\text { Parameter } \\
\text { Estimate }\end{array}$ & $\begin{array}{r}\text { Std. } \\
\text { Error }\end{array}$ & t-value \\
\hline Rat & & & \\
\hline Intercept & 0.17 & 0.08 & 2.02 \\
Rat-dummy & $0.64^{* *}$ & 0.29 & 2.24 \\
$R^{2}$ & 0.1005 & & \\
f-value & 5.03 & & \\
p-value & 0.0299 & & \\
\hline \multicolumn{5}{c}{} \\
\hline Snake & & & \\
\hline Intercept & 0.25 & 0.09 & 2.91 \\
Snake-dummy & -0.41 & 0.34 & -1.2 \\
$R^{2}$ & 0.0310 & & \\
f-value & 1.44 & & \\
p-value & 0.2366 & & \\
\hline
\end{tabular}

To control for major macroeconomic factors such as GDP and Unemployment Rate we also tried the following regression for HSI.

$H P R_{\text {Hang Seng }}=\alpha+\beta$ (animal_year_dummy) $+\gamma($ annual \% change in GDP $)+\lambda($ annual \% change in Unemployment Rate) 


\section{Macrothink}

The OLS results for both Rat and Snake dummies indicate that the annual \% change in Unemployment Rate explains most of variation in returns (at $1 \%$ significant level). The GDP and the dummy (Rat and Snake) coefficients are not significant. For brevity, tabulated results are not reported here.

To further investigate, in Table 7, we test the significance of the difference between the means (t-test) and the medians (Wilcoxon-Mann-Whitney test) of a one animal year versus the rest. We repeat this process for each of the 12 animals. Table 7 presents the results only for the Rat and the Snake years.

Table 7. Return Comparison Rat, Snake versus Others

\begin{tabular}{lrr}
\hline Rat & Mean & $\mathrm{N}$ \\
\hline Yes & 0.8130 & 4 \\
No & 0.1693 & 43 \\
Mean difference & 0.6437 & \\
$p$-value & 0.0299 & \\
Median & & \\
one-sided p-value & 0.4824 & \\
two-sided p-value & 0.9649 & \\
\hline Snake & Mean & $\mathrm{N}$ \\
\hline Yes & -0.1579 & 3 \\
No & 0.2501 & 44 \\
Mean difference & -0.408 & \\
$p$-value & 0.0061 & \\
Median & & \\
one-sided $p$-value & 0.0415 & \\
two-sided p-value & 0.0830 & \\
\hline
\end{tabular}

Based on the univariate analysis shown in Table 7, Rat has significantly higher mean and Snake year significantly lower mean and median compared to the rest of the animal years. Other years are not presented as no statistical significance was observed. When we repeat this analysis for S\&P, only Snake and Rooster years show lower HPRs than the rest. The statistical significance is $10 \%$ (5\%) for Snake (Rooster). Rooster also shows significantly lower median HPRs. Results are not reported here.

\section{Summary}

Previous studies show how mood, character, attitude, and daily experience of individuals affect stock market returns. In the same vein, it is documented that holidays, religious feasts, and cultural beliefs affects stock market returns. Since ancient times, Chinese zodiac culture and astrology has affected the way people in Asian and occasionally in western societies make minor or major decisions. Each zodiac year is distinguished by a symbolic animal or an 


\section{Macrothink}

element. In this paper we investigate whether or not the HSI returns were affected by the demeanor of a specific zodiac year -animal or element. When data is broken by the animal symbols, the Rat year shows the highest gain and the Snake year displays the biggest lose consistent with common belief in the Chinese culture. We do not find statistical significance for the remaining animal-years. For S\&P, both Snake and Rooster show lower holding period returns.

We also document that the yang years show higher returns for both HSI and S\&P. Water and Earth years and more specifically, yang Water and yin Earth years display relatively higher returns. Even though the results suggest a difference, the statistical tests come short of finding definitive difference in returns, possibly due to small sample size.

\section{References}

Ariel, R.A. (1990). High Stock Returns before Holidays: Existence and Evidence on Possible Causes. Journal of Finance, 1611-1626. http://dx.doi.org/10.1111/j.1540-6261.1990.tb03731.x

Hirshleifer, D., \& Shumway, T. (2003). Good Day Sunshine: Stock Returns and the Weather. Journal of Finance, 58, 1009-1032. http://dx.doi.org/10.1111/1540-6261.00556

Mehran, J., Meisami, A., \& Busenbark, J.R. (2012). L'Chaim: Jewish holidays and stock market returns. Managerial Finance, 38, 641-652. http://dx.doi.org/10.1108/03074351211233104

Saunders, E.M. (1993). Stock Prices and Wall Street Weather. The American Economic Review, 83, 1337-1345.

Sullivan, R., Timmermann, A., \& White, H. (2001). Dangers of data mining: The case of calendar effects in stock returns. Journal of Econometrics, 105, 249-286. http://dx.doi.org/10.1016/S0304-4076(01)00077-X

Yuan, K., Zheng, L., \& Zhu, Q. (2006). Are investors Moonstruck? Lunar phases and stock $\begin{array}{lllll}\text { returns. Journal of } & \text { Empirical }\end{array}$ http://dx.doi.org/10.1016/j.jempfin.2005.06.001 